\title{
SURGERY OF THE THYROID GLAND
}

\author{
Jan Vokurka, Stanislava Jakoubková, Jaroslav Rüžička
}

Department of Otorhinolaryngology, Head and Neck Surgery, Charles University, Faculty of Medicine, Hradec Králové; (Head: doc. MUDr. J. Vokurka, CSc.)

Summary: Since the year 1987 to 1996 all kinds of thyroid surgeries were performed at the ENT Department. Altogether 604 patients underwent 655 surgeries. Total lobectomy or total thyroidectomy represented the most common procedures. Due to a gentle surgical technique, which we call „preparation“, good results were achieved in the morbidity of laryngeal recurrent nerve (permanent palsy in $0,6 \%)$, and in perioperative or postoperative bleeding $(0,3 \%$ of wound revisions, $0,5 \%$ of blood transfusions). Postoperative hypoparythyroidism was found in $4,7 \%$ patients. There is no correlation between postoperative hoarseness and laryngeal recurrent nerve palsy. Laryngeal endoscopy immediately after surgery is the most valuable diagnostic procedure.

Key words: Thyroidectomy; Surgical technique; Complications of thyroidectomy

\section{Introduction}

Thyroid gland surgery was performed at our Department only occasionally until the year 1987 . The patients treated for postoperative recurrent nerve palsy at the Department of Phoniatry stimulated our interest in the possibilities how to minimize their numbers. Since November 1987 all types of thyroid gland surgery were adopted step by step in our Department. We have started with unilateral lobectomy mostly for adenomas or cysts, later we have performed total thyroidectomy for cancer and even later for some cases of thyreotoxicoses of Graves-Basedow's autoimmune type. The number of operated patients rose every year (Table 1). Our surgical strategy is being based on:

- Operation should be sufficient for treatment of the disease (complete removal of the lesion)

- The extent of the surgery should avoid the need to return to the previously operated area in a future case of disease recurrence when reoperation is mandatory (9).

Operations on the thyroid were performed to reduce its function (thyrotoxicosis), reduce its volume (non-toxic goitre), or treat malignancy. It is essential for the surgeon to be familiar with the anatomy of the thyroid and parathyroid glands. Acquaintance with the blood and nerve supplies to the larynx and their anatomic variations is necessary for a safe thyroid surgery. Anatomical study and training of the surgical procedure at the Department of Anatomy is recommendable. Extremely close cooperation with an endocrinologist in the indication, preoperative preparation and selection of the optimal method and postoperative care is essential $(5,6)$.

\section{Methods}

Between November 1987 and the end of December 1996 a total of 604 patients underwent different types of thyroid surgery (Table 1.). We record a constant yearly increase of surgeries. Altogether there were performed 655 operations.

Table 1: Thyroid gland surgery

\begin{tabular}{|c|c|r|r|}
\hline Year & Females & Males & Total \\
1988 & 15 & 1 & $\mathbf{1 6}$ \\
\hline 1989 & 25 & 3 & $\mathbf{2 8}$ \\
\hline 1990 & 42 & 3 & $\mathbf{4 5}$ \\
\hline 1991 & 36 & 3 & $\mathbf{3 9}$ \\
\hline 1992 & 41 & 5 & $\mathbf{4 6}$ \\
\hline 1993 & 59 & 8 & $\mathbf{6 7}$ \\
\hline 1994 & 65 & 6 & $\mathbf{7 1}$ \\
\hline 1995 & 108 & 16 & $\mathbf{1 2 4}$ \\
\hline 1996 & 138 & 30 & $\mathbf{1 6 8}$ \\
\hline TOTAL & $\mathbf{5 2 9}$ & $\mathbf{7 5}$ & $\mathbf{6 0 4}$ \\
\hline
\end{tabular}

\section{Preoperative preparation}

Most of the patients are referred for surgery by the endocrinologist. Biochemical tests include estimation of se- 
rum thyroxin (T3,T4), serum calcium, TSH, and thyroid antibodies, are routinely performed. Also the preoperative $\mathrm{X}$ rays of the thorax is performed regularly, CT or MRI is done only in selected patients where technical or surgical problems are to be expected. Scanning with radio-isotopes is used in patients with suspected „hot nodule“ or with nodular goitre. Sonography was performed in all patients. This examination is essential today and completes the preoperative assessment. Ultrasound is of a great value in distinguishing cystic from solid lesions. Fine needle biopsy (cytology) is used in cases of nodular lesions due to its high sensibility to detect malignancies. Estimation of the calcitonin level is done in patients with medullary carcinoma and in all their relatives.

Special preoperative medication is used in thyreotoxicosis only. Carbimazol is being administered to achieve euthyroid state. Iodine (Lugols solution, sodium iodine) is being used for 10-14 preoperative days to reduce vascularity and friability of the gland. No preoperative medication is given in patients with suspicion of carcinoma, especially in patients with nodular goitre.

\section{The operation}

All operations are performed under general endotracheal anesthesia in supine position. The neck is extended by placing a sandbag under the shoulders. The upper part of the table is raised to reduce tissue congestion. Local infiltration of Xylocaine with epinephrine is used to reduce bleeding of the skin incision.

\section{Thyroid lobectomy}

Total lobectomy is the most frequent surgery on the thyroid gland (Table 2). This is performed in patients with solitary nodules. The skin incision extends from one sternomastoid muscle to the opposite one, two fingers above the sternal notch. To divide the platysma electrocautery is used. The upper and lower skin flaps are then elevated. The fascia over the strap muscles is incised in the midline and the strap muscles are retracted laterally.

Horizontal incision of the sternohyoid muscle is used in cases of large thyroid glands. We use mainly the lateral approach based on mobilization of the lobe from the lateral side. The middle thyroid vein is interrupted when found. Superior pole vessels are transsected, as well as the inferior vein and lobe is thus mobilized and delivered into the wound. The last step is represented by dissection and mobilization of the posterior surface of the lobe with localization of the recurrent laryngeal nerve, parathyroid glands and their blood supply. After dissection of the parathyroid glands with their blood supply intact, all terminal branches of the inferior thyroid artery are transsected. Care should be taken in the region of the tracheo-esophageal groove to identify the recurrent laryngeal nerve. Transsection of the thick fascial suspension of the lobe to the cricothyroid membrane (Berry's ligament) is the last step of the procedure. This posterior suspensory ligament should be cut carefully, under direct vision. Recurrent laryngeal nerve is kept in view all the time. We prefer its visual identification but sometimes this is not possible. In these cases we perform dissection very close to the surface of the lobe or we incise the capsule and continue subcapsularly. Berry's ligament is an important structure in relation to the recurrent laryngeal nerve. Lore considered this ligament as one of the most important structures in thyroidectomy (3). The medial approach, as described by Loré, we use mostly in smaller lesions, but generally less frequently than the lateral one. After transsection of the isthmus and superior displacement of the inferior pole of the lobe, its posterior sus pensory ligament is divided and the recurrent laryngeal nerve is identified. We carry out meticulous dissection using delicate retractors, dissectors and Deschampess needles for ligation of the vessels prior to their transsection. This procedure we call „preparation technique“. Such a technique consumes more time than the conventional one, but offers better results in preventing laryngeal recurrent nerve palsy or damage of the parathyroid gland vessels. We avoid rough handling and inadvertent clamping tissues during the dissection and then transsection of the tissue between hemostats. This technique we call „resection“. We have had an opportunity to use the nerve stimulator for easier identification of the laryngeal recurrence nerve. This device is helpful but not in each case.

The operation technique of the total lobectomy is grounding for other surgeries.

\section{Total thyroidectomy}

After the first side completed the same procedure is performed on the opposite side. If it is possible we start usually on the left side and remove all parts of the thyroid in one block.

In case of some difficulties we start with transsection of the isthmus and each lobe is dissected separately. Subtotal thyroidectomy is performed by leaving a small remnant of the tissue but with the same technique as a total procedure. We use the same nomenclature as Dvořák (2).

\section{Subtotal thyroidectomy}

When resecting nodular goitre, the margin of the remnant is given by the normal tissue. Most conventional are remnants of the posterior parts at the both sides. Nevertheless, sticking to our surgical rule „not to return to the previously operated area" we rather perform a total lobectomy on one side and subtotal on the other. In case of recurrence, reoperation is performed on the latter side. Otherwise, we leave adequate remnants of the superior parts of both lobes connected with the superior thyroid vessels. 


\section{Retrosternal goitre}

Retrosternally positioned goitres are usually easily shifted in the neck by traction and gentle dissection from above, once the vessels have been secured. In cases of excessive size of retrosternal protuberations, cooperation with the thoracic surgeon is necessary.

Once haemostasis is satisfactory, the neck can be closed. We use the suction Redon drain (for 24, 48 or 72 hours, depending on the amount of blood suctioned; collection of $10 \mathrm{ml}$ or less indicates the drain removal). The muscles are approximated in the mid-line with interrupted or continuous absorbable sutures. The skin is usually sutured with intradermal continuous suture. Laryngoscopy is performed at the end of the surgery immediately after endotracheal tube removal. We use McIntosh laryngoscope, in difficult cases there are better results with a 25 degree, $2.7 \mathrm{~mm}$ endoscope (Richard Wolf, Knittlingen, Germany). Also a fibrolaryngoscope may be used.

\section{Results}

In 604 patients who underwent different types of thyroid gland surgery, 655 operations were performed. The female to male ratio was $7: 1$. Reoperations were done in patients with preoperative diagnosis of benign lesion (nodular type mostly), but with postoperative final diagnosis of carcinoma. This secondary completion of the total thyroidectomy had the same morbidity as the primarily performed total thyroidectomies (1).

Hemorrhage is usually peroperative or within a short period of time postoperatively. The wound had to be reopened early after operation in two patients $(0,3 \%)$. Reoperations revealed the source - the superior thyroidal artery in both cases. Blood transfusion was needed in 4 patients $(0,5 \%)$. Hemorrhage during operation is minimized by a gentle dissection technique using a bipolar coagulation and dividing vessels between ligatures (Table 2).

\section{Hypoparathyroidism}

The serum calcium is usually low after the total thyroidectomy even if all parathyroid glands were saved. Hypocalcemia in these cases is present mostly without a clinical manifestation. This hypocalcemia is transient due to blood supply damage, not to the parathyroid gland removal. Transient hypocalcemia was found in 43 patients. Treatment according to symptoms rather than estimation of serum calcium was applied in 36 patients (by calcium supply in 11 patients and in 25 of them dihydrotachysterol was added) (Table 2). Treatment lasting for more than 1 year very likely proves a permanent hypoparathyroidism (14 patients).

\section{Recurrent Laryngeal Nerve Palsy}

Laryngeal nerve is at risk of damage in total thyroidectomy on both sides, as well as in the subtotal thyroidecto- my. In the subtotal thyroidectomy the risk is different, in the classic procedure (2) there exists the risk on both sides, but smaller one when compared with total thyroidectomy. In our modification with one-sided total and the other-sided partial procedure, only the side with total lobectomy is at risk. In the modification with remnants of tissue left on the superior thyroidal vessels, both laryngeal recurrent nerves are at risk. Partial thyroidectomy should lack the risk totally. In 604 patients with 655 operations, 961 laryngeal recurrent nerves were at risk of damage (Table 2). Postoperative laryngoscopy was impossible in the usual way in 15 patients $(2,4 \%)$ only. Immobile vocal cords or evident discoordination of vocal cords movement were revealed in 102 cases $(10,6 \%)$ from 961 cases, where laryngeal recurrent nerve was at risk (100\%). From this group of patients the recurrent nerve palsy was evident in magnifying laryngoscopy the day after operation in 92 cases $(9,6 \%)$. In three patients palsy has not been recognized immediately after operation, but as late as the next day. From 102 cases of laryngeal recurrent nerve palsy recognized immediately after the operation, 6 patients suffered for more then 6 months and are considered to have a permanent palsy $(0,6 \%)$. We compared the number of palsies and number of patients suffering from hoarseness after operation (Table 3). Hoarseness was more often then palsy. There were 242 patient who suffered from hoarseness following thyroid gland surgery, while only 102 patients had a laryngeal recurrent nerve palsy. Palsy could be connected with hoarseness (70 cases) but not in each case, 32 patients with palsy were without voice impairment. Postoperative excavated vocal cord on one side of the larynx which confirms superior laryngeal nerve palsy was diagnosed in 12 patients $(1,2 \%)$.

Table 2: Postoperative complications

\begin{tabular}{|l|r|c|}
\hline Complication & No & $\%$ \\
\hline Hemorrhage: patients at risk & $\mathbf{6 0 5}$ & $\mathbf{1 0 0}$ \\
\hline Postop. revision needed & $\mathbf{2}$ & $\mathbf{0 , 3}$ \\
\hline Blood transfusion & $\mathbf{3}$ & $\mathbf{0 , 5}$ \\
\hline Parathyroid glands at risk & $\mathbf{2 9 8}$ & $\mathbf{1 0 0}$ \\
\hline Hypoparathyroidism temporary & $\mathbf{4 3}$ & $\mathbf{1 4 , 4}$ \\
\hline Hypoparathyroidism permanent & $\mathbf{1 4}$ & $\mathbf{4 , 7}$ \\
\hline Recurrent nerve at risk & $\mathbf{9 6 1}$ & $\mathbf{1 0 0}$ \\
\hline Vocal cord preoperative & $\mathbf{1}$ & $\mathbf{0 , 1}$ \\
\hline Palsy postoperative & $\mathbf{1 0 2}$ & $\mathbf{1 0 , 6}$ \\
\hline Temporary & $\mathbf{9 0}$ & $\mathbf{9 , 4}$ \\
\hline Permanent & $\mathbf{6}$ & $\mathbf{0 , 6}$ \\
\hline
\end{tabular}

Table 3: Hoarseness and recurrent nerve palsy

\begin{tabular}{|l|r|r|r|}
\hline & Palsy & Hoarseness & Total \\
\hline Hoarseness & $\mathbf{7 0}$ & $\mathbf{1 7 2}$ & $\mathbf{2 4 2}$ \\
\hline Palsy & $\mathbf{3 2}$ & $\mathbf{7 0}$ & $\mathbf{1 0 2}$ \\
\hline Total & $\mathbf{1 0 2}$ & $\mathbf{2 4 2}$ & \\
\hline
\end{tabular}




\section{Postoperative care}

After the operation the patient is placed in the recovery unit until fully conscious. The patients after total thyroidectomy, especially due to thyrotoxicosis, stay at the unit until the next morning, exceptionally longer.

The suction drain (Redon) is removed between $24 \mathrm{hrs}$ and 72 hrs, usually after 48 hrs. Thereafter a light dressing may be used to cover the incision. The intradermal stitch is removed usually on the 7 th postoperative day. The patient is sent back to be seen by the endocrinologist.

\section{Discussion}

The operation technique is based on the conception of total surgery (8), cooperation with an endocrinologist is mandatory. As to the radicality of the procedure we state two rules: 1.- surgery should be fully sufficient for the treatment or removal of a lesion and 2.- must be extensive enough to avoid the need to a future return to the previously operated area if recurrence of the disease occurs and reoperation is necessary. For these reasons we commonly perform total lobectomy or total thyroidectomy. Other indication for the total thyroidectomy are some GravesBasedows thyrotoxicoses and most of the thyroid papilocarcinomas (with iodine accumulation).

Radioactive Iodine-131 therapy is beneficial in those patients whose primary tumors or metastatic deposits concentrate radioactive iodine. After total removal of the thyroid gland the patient develops hypothyroidism and the concentration of the iodine in the thyroid tissue remnants, including metastases, is more effective. Thus the total thyroidectomy or completion of total thyroidectomy (1), plays an important role in our surgical policy (7). This radical therapy represents the standard in Czech Republic and excellent results advocate this procedure which is not generally accepted everywhere, but in the USA (4).

Our results, as go for permanent postoperative recurrent nerve palsy and perioperative bleeding, are comparable or better than data in literature $(2,7)$. The gentle dissection technique using small dissectors, special forceps for bipolar coagulation, and the ligation of vessels prior to their transsection are most probably the main reasons. Furthermore, we separate the branches of the inferior thyroid artery in the thyroid gland capsule, not in its trunk.

The recurrent nerve is being identified and displayed. This dissection procedure is time consuming but perioperative bleeding and especially the damage of the nerve can be thus minimized. Direct laryngeal endoscopy either with Mc Intosh laryngoscope or with fibrolaryngoscope immediately after removing endotracheal tube is very useful, mainly in patients after the total thyroidectomy. Endoscopy shows normal or pathological mobility of the vocal cords and in case of palsy we are able to start proper treatment for the safe postoperative recovery.
Hoarseness is highly unreliable for estimation of recurrent nerve palsy. Unilateral palsy is often asymptomatic and discovered only by laryngoscopy. On the other hand, hoarseness could be due to intubation with normal vocal cords mobility.

\section{Conclusion}

We conclude that thyroid gland surgery is a part of the neck surgery which needs a very gentle surgical technique. This technique we call „preparation“, in contradiction to „resection“ technique. Preparation procedure is time consuming but the results are better as far as the postoperative recurrent nerve palsy, hypoparathyroidism or perioperative bleeding are concerned. The surgeon should be familiar with the neck surgery, of course. Thyroid operations should not be available for the occasional surgeon.

\section{References}

1. Calabro S. et al. Morbidity of completion thyroidectomy for initially misdiagnosed thyroid carcinoma. Head Neck Surg 1988:10:235-8.

2. Dvořák J. Surgical Anatomy and Technique of Thyreoid Surgery (Czech). Edited by Dům medicíny:Praha, 1995:1238.

3. Lore JM. Practical anatomical considerations in thyroid tumour surgery. Arch Otolaryngol 1983:109:568-74.

4. Mazzaferri EL, Young RL. Papillary thyroid carcinoma: A ten year follow-up report of the impact therapy in 576 patients. Am J Med 1981:70:511-8.

5. Nahodil V. Surgery of the thyroid gland (Czech). Avicenum:Praha, 1989.

6. Němec $J$ et al. Indications of the thyroid gland operation and preoperative preparation of the patients ( $\mathrm{Czech})$. Prakt Lek 1987:67:429-32.

7. Perzik S. The place of total thyroidectomy in the management of 909 patients with thyroid disease. Am J Surg 1976,132:480-3.

8. Vokurka J, Jakoubková J, Hybášek I. Functional surgery of thyroid gland at E.N.T. Department in Hradec Králové (Czech). Cesk Otorinolaryngol Foniatr 1992,41:257-61.

9. Vokurka J, Jakoubková S. Thyroidectomy. Cesk Otorinolaryngol Foniatr 1993,42:127-9.

Submitted April 1997.

Accepted September 1997.

Doc. MUDr. Jan Vokurka, CSc., Department of Otorhinolaryngology, Head and Neck Surgery, Charles University, Faculty of Medicine, 50005 Hradec Králové, Czech Republic. 